DOE/ER/40561--097

DE93011009

\title{
Time-Reversal-Noninvariant, Parity-Conserving Nuclear Interactions
}

\author{
Wick C. Haxton 1,2 and Antje Höring1 \\ IInstitute for Nuclear Theory, HN-12, and Department of Physics, FM-15, \\ University of Washington, Seattle, WA 98195
}

${ }^{2}$ Department of Physics, University of Melbourne, Parkville 3052, AUSTRALIA

\section{DISCLAIMER}

\begin{abstract}
This report was prepared as an account of work sponsored by an agency of the United States Government. Neither the United States Government nor any agency thereof, nor any of their employees, makes any warranty, express or implied, or assumes any legal liability or responsibility for the accuracy, completeness, or usefulness of any information, apparatus, product, or process disclosed, or represents that its use would not infringe privately owned rights. Reference herein to any specific commercial product, process, or service by trade name, trademark, manufacturer, or otherwise does not necessarily constitute or imply its endorsement, recommendation, or favoring by the United States Government or any agency thereof. The views and opinions of authors expressed herein do not necessarily state or reflect those of the United States Government or any agency thereof.
\end{abstract}

\section{PREPARED FOR THE U.S. DEPARTMENT OF ENERGY UNDER GRANT DE-FG06-90ER40561}

This report was prepared as an account of work sponsored by the United States Government. Neither the United States nor any agency thereof, nor any of their employees, makes any warranty, express or implied, or assumes any legal liability or responsibility for the accuracy, completeness, or usefulness of any information, apparatus, product, or process disclosed, or represents that its use would not infringe privately owned rights. Reference herein to any specific commercial product, process, or service by trade name, mark, manufacturer, or otherwise, does not necessarily constitute or imply its endorsement, recommendation, or favoring by the United States Government or any agency thereof. The views and opinions of authors expressed herein do not necessarily state or reflect those of the United States Government or any agency thereof.

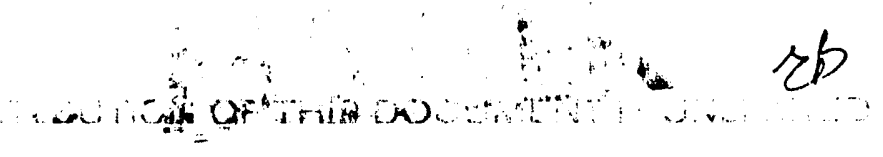




\title{
TIME-REVERSAL-NONINVARIANT, PARITY-CONSERVING NUCLEAR INTERACTIONS
}

\author{
W. C. HAXTON ${ }^{1,2}$ and A. HÖRING ${ }^{1}$ \\ ${ }^{1}$ Insiitute for Nuclear Theory HN-12 and Department of Physics FM-15 \\ University of Washington, Seattle, WA 98195 \\ and \\ ${ }^{2}$ Department of Physics, University of Melbourne, Parkville 3052, Australia
}

\begin{abstract}
We review the work that has been done in compound nucleus studies of timereversal-noninvariant (TRNI), parity-conserving (PC) $N N$ interactions. Following Simonius and others, we also discuss the general form of TRNI PC meson-exchange potentials that can be constrained by such studies. We then explore the competing limits that can be obtained from atomic electric dipole moments produced by weak radiative corrections to long-ranged TRNI PNC interactions. Both classes of constraints limit the magnitude of typical TRNI PC matrix elements to about $10^{-3}$ of those for strong interactions. We contrast these limits to those recently obtained from short-ranged oneand two-loop weak radiative corrections.
\end{abstract}




\section{Introduction}

Time-reversal-noninvariant (TRNI), parity-nonconserving (PNC) interactions arise naturally in the standard model through the Kobayashi-Maskawa phase in the threegeneration quark mass matrix and through the $\theta$ term. New possibilities arise in extensions of the standard model with left-right symmetry, extended Higgs sectors, etc. ${ }^{1}$ ) Paity-conserving (PC) TRNI interactions are much more exotic in gauge models. In fact, it has been argued that, in renormalizable gauge models with elementary quarks, flavor-conserving TRNI PC interactions between quarks arise only in second order in the meson-boson couplings, e.g., through weak corrections to TRNI PNC interactions ${ }^{2}$ ). Current limits on neutron and atomic electric dipole moments, combined with estimates of weak radiative corrections, then suggest that such interactions might be characterized by couplings $\sim 10^{-16}$.

Yet one can take a more pragmatic view, asking what experiments directly constrain TRNI PC nucleon-nucleon interactions. Existing bounds are far above the $10^{-16}$ level. Some of the most stringent direct tests of such interactions have been made, following a suggestion by Wigner ${ }^{3}$ ), in compound nucleus studies of detailed balance and nuclear energy level fluctuations. Limits are frequently quoted in terms of the typical strenigth of TRNI PC nuclear matrix elements to those of the residual strong interaction, $\alpha \lesssim$ $2 \cdot 10^{-3}$. As Hans Weidenmüller and his collaborators have played a central role in developing the statistical theory of compound nucleus reactions and generalizing it for studies of TRNI, we felt this topic and related tests of TRNI would be an appropriate theme for our contribution to this Festschrift.

Another set of important experimental constraints comes from observables that are TRNI and PNC, but may arise from weak corrections to TRNI PC interactions ${ }^{4}$ ). For instance, neutron and atomic electric dipole moment searches, which have reached extraordinary levels of precision, can be used to limit TRNI PC interactions. Dimen- 
sional arguments suggest such limits constrain TRNI PC meson-nucleon couplings to $\left.\lesssim 10^{-4} \cdot 1,4\right)$

In this paper we hope to better quantify the relationship between compound nucleus (CN) and electric dipole moment (edm) constraints on long-ranged TRNI PC interactions. We begin by reviewing the work that has been done in compound nuclei. In the second section, following Simonius ${ }^{5}$ ) and $\mathrm{Herczeg}^{6}$ ), we consider the general form of the TRNI PC interaction in meson exchange models. In the third section we discuss one mechanism for generating an atomic edm, a TRNI PC nuclear interaction mediate by $\rho$ exchange coupled with $Z$ exchange between atornic electrons and the nucleus. While a variety of other mechanisms can similarly generate edms from TRNI PC interactions ${ }^{7}$ ), this example has some interesting experimental consequences. The limits we extract are then translated into a constraint on $\alpha$, the ratio of typical TRNI and TRI $N N$ matrix elements. We conclude that such atomic edm limits on TRNI PC interactions are at least comparable to those obtained in the best $\mathrm{CN}$ studies. These limits from long-distance contributions to edms are then compared to the stringent bounds obtained recently by Conti and Khriplovich from studies of short-ranged mechanisms.

\section{Constraints from Compound Nuclei}

TRNI PC interactions can be tested directly in detailed balance experiments in the region of overlapping compound nucleus resonances. The observable is $X=1-\left|R_{a b}\right|^{2}$ where

$$
\left|R_{a b}\right|^{2}=\frac{\left.\left\langle\sigma_{a b} \sigma_{b a}\right\rangle-<\sigma_{a b}\right\rangle\left\langle\sigma_{b a}\right\rangle}{\left\langle\sigma_{a b}><\sigma_{b a}>\right.}
$$

measures the correlation between the cross section $\sigma_{a b}$ of a $\mathrm{CN}$ reaction from channel $a$ to $b, a \neq b$, and that of the inverse reaction, $\sigma_{b a}$. The angular brackets $<>$ denote an average over a suitable energy interval. The best upper limit that has been obtained is $\left.|X|<10^{-6} \cdot{ }^{8}\right)$ 
The problem of relating $X$ to the TRNI part of the nuclear Hamiltonian was studied by Boosé, Harney, and Weidenmüller ${ }^{9}$ ). The statistical $S$ matrix ${ }^{1}$ ) describing $\mathrm{CN}$ reactions is represented by an $N$-level Breit-Wigner

$$
\begin{aligned}
S_{a b}(E) & =\delta_{a b}-2 i \pi \sum_{\mu \nu} W_{a \mu}\left(D^{-1}\right)_{\mu \nu} W_{\nu b} \\
D_{\mu \nu} & =\left[\left(E \delta_{\mu \nu}-H_{\mu \nu}+i \pi \sum_{c} W_{\mu c} W_{\nu c}\right]\right.
\end{aligned}
$$

where $N$ is the number of $\mathrm{CN}$ levels, modeled as orthonormal bound states and labeled by the indices $\mu, \nu \ldots$ The open channels, numbering $\Lambda$, are denoted $a, b, c, \ldots$ The levels are coupled to each other by matrix elements of the nuclear Hamiltonian, $H_{\mu \nu}=H_{\nu \mu}^{*}$, and to the channels by the matrix elements $W_{a \mu}=W_{\mu a}^{*}$. While both sets of matrix elements can be taken to be real if the theory is TRI, TRNI forces them to be complex.

The statistical properties of the $S$ matrix follow from those of the Hamiltonian $H$, which Boosé et al. modeled from random matrix theory. The Hamiltonian was decomposed into two parts

$$
H=H^{(o)}+H^{(u)}
$$

where the (dominant) contribution $H^{(o)}$ is TRI and is drawn from a Gaussian orthogonal ensemble (GOE), while the TRNI matrix $H^{(u)}$ is drawn from a Gaussian unitary ensemble (GUE). $H^{(o)}$ and $H^{(u)}$ are assumed to be uncorrelated, to have zero mean values, and to have second moments in the ratio $1: N^{-1} \alpha_{0}^{2}$. Boosé et al. then calculated $\left|R_{a b}\right|^{2}$ to leading order in $\alpha_{0}^{2}$ for the case of strongly overlapping $\mathrm{CN}$ resonances and in the limit $\Lambda \gg 1$. They found $R_{a b}$ could be expressed as the ratio of two two-point functions, with the energy averages of Eq. (1) replaced by appropriate ensemble averages, under the assumption of ergodicity. An approximate evaluation of this expression led the authors to conclude that $R$ was independent of the channel indices and dependent on $\alpha_{0}^{2}$ and the sum of the channel transmission coefficients. After taking the total 
CN spreading width from experiment and the Fermi gas level density of Gilbert and Cameron, a comparison with the experimental limit on $X$ yielded $^{9}$ )

$$
\alpha_{0}^{2} \lesssim 7.3 \cdot 10^{-6}
$$

with $\left|\alpha_{0}\right|$ and $N$ then providing a measure of the strength of the TRNI part of $H$ relative to the TRI part. Boosé et al. also showed that the deviation of $R$ from unity could be expressed as the ratio of the spreading widths for $H^{(u)}$ and $H^{(0)}$, and suggested that this might be the more useful parameterization, exhibiting less dependence on the choice of $E$ and mass number $A$.

Related CN tests of TRNI were performed by the Rochester group, who studied spectral fluctuations and strength distributions in the regime of isolated resonances ${ }^{10}$ ). The spectral sensitivity to TRNI arises from the fact that the von Neumann-Wigner level repulsion, and the more general Dyson-Mehta spectral rigidity, are stronger for a TRNI Hamiltonian than for a TRI one. The strength distribution sensitivity arises because a TRI Hamiltonian generates a Porter-Thomas distribution ( $\chi_{1}^{2}$ form) while a maximally TRNI Hamiltonian produces a $\chi_{2}^{2}$ distribution. Resonance widths are more difficult to extract from experiment and thus more difficult to analyze. For this reason we will limit our discussion to the spectral fluctuations, while noting that the authors of Ref. 10 did consider the consistency of both data sets.

French et al. decomposed the Hamiltonian into TRI and TRNI pieces, in a manner similar to Eq. (3),

$$
H(\alpha)=H^{S}+i \alpha H^{A}
$$

with $H^{S}$ and $H^{A}$ symmetric and antisymmetric matrices, respectively, and with $\alpha$ governing the relative strength of TRNI. The matrix elements $H_{i j}, i \leq j$, were chosen independently from zero-centered Gaussian distributions with variances $\sigma_{i j}^{2}=v^{2}\left(1+\delta_{i j}\right)$ and $v^{2}\left(1-\delta_{i j}\right)$, respectively, for $H^{S}$ and $H^{A} . H^{S}$ is a member of the GOE, while the 
TRNI component $H^{A}$ is a member of the GUE. The natural parameter that describes the transition from GOE to GUE is

$$
\Lambda(\alpha, E)=\left|i \alpha H_{i j}^{A}\right|^{2} / D^{2}(E)=\alpha^{2} v^{2} / D^{2}(E),
$$

where $D(E)$ is the average level spacing at an excitation energy $E$. The goal of the analysis is to find, from fluctuation theory and nuclear data, the maximum permissible value of $\Lambda$.

The data set used by the Rochester group included 1702 levels in 32 nuclei $\left.^{11}\right)$. The optimal measure for restricting $\Lambda$ proved to be ${ }^{11}$ ) the variance of the number of levels found in an interval which, on the average, contains one level. Depending on how the data from different nuclei were grouped into subsets of comparable level density, upper bounds on $\Lambda$ were obtained in the range $0.1<\Lambda^{1 / 2}<0.2$. To improve these bounds by an order of magnitude the data set would have to be enlarged to about $10^{6}$ levels.

Nontrivial tasks remain: the extraction of $\alpha$ from Eq. 5 as well as the interpretation of $\alpha$ in terms of the strength of typical residual $N N$ interactions. These tasks cannot be attacked with conventional shell model tools because the appropriate Hilbert spaces are enormous. French and his collaborators instead exploited spectral averaging techniques to make these connections, as described in Ref. [12]. The analysis yielded $|\alpha| \lesssim 2$. $10^{-3}$ (99\% c.1.), and the interpretation of $\alpha$ as the ratio of typical TRNI NN matrix elements to those of the TRI residual shell-model interaction. The limit at $80 \%$ c.l. is $1.3 \cdot 10^{-3}$. A corresponding analysis ${ }^{12}$ ) of the detailed balance limits of Boosé, Harney, and Weidenmüller $\left.{ }^{9}\right)$ gave $4 \cdot 10^{-3}(99 \%$ c.l. $)$ and $0.2 \cdot 10^{-3}(80 \%$ c.l. $)$, illustrating how critically this result depends on the statistical confidence one requires.

One other possible TRNI PC observable in the regime of isolated CN resonances exploits the five-fold correlation ${ }^{13,14}$ )

$$
\vec{\sigma} \cdot(\vec{k} \times \vec{I})(\vec{k} \cdot \vec{I})
$$


where $\vec{\sigma}$ is the neutron spin, $\vec{k}$ the neutron momentum, and $\vec{I}$ the nuclear spin. $\mathrm{Kabir}^{13}$ ) suggested measuring the neutron transmission asymmetry for the two polarization directions perpendicular to the $\vec{k} \times \vec{I}$ plane. (This is not technically a test of TRNI unless the target is invariant under a 180 degree rotation through a central axis along $\vec{k} \times \vec{I}$.) The effects of TRNI in the neutron-target interactions can be greatly enhanced in systems with two close-lying resonances $\left.{ }^{15}\right)$. The relation between the asymmetry and the TRNI interaction has been explored by Barabanov ${ }^{16}$ ), by Bunakov and Gudkov ${ }^{17}$ ), and by Bunakov, Davis, and Weidenmüller ${ }^{18}$ ). A measurement with epithermal neutrons to exploit possible CN enhancements has not yet been attempted. There are technical hurdles associated with the need to analyze the spin of the transported neutrons ${ }^{19}$ ). However a transmission experiment for higher energy ( $2 \mathrm{MeV})$ neutrons through a cryogenically aligned ${ }^{165} \mathrm{Ho}$ target has been successfully completed, producing the limit $\left.|\alpha| \lesssim 5 \cdot 10^{-3} \cdot{ }^{20}\right)$ It is likely that this limit could be improved substantially by employing a more intense polarized neutron source.

\section{The TRNI $N N$ Interaction}

The possible structure of a TRNI PC $N N$ interaction has been known for some time. Herczeg ${ }^{6}$ ) specified the most general spin and isospin structure of such interactions. Simonius considered the most general form for TRNI PC meson-exchange potentials, thereby specifying the radial dependence of the interaction. The lightest possible exchange, $\rho^{ \pm}$, will be used as a representative potential in the next section.

Simonius's conclusions about TRNI PC meson-exchange potentials for on-shell nucleons were precisely stated:

1) A scalar exchange $(J=0)$ cannot generate a TRNI PC $N N$ potential. Thus there is no pion-range interaction.

2) For $J \neq 0$ normal parity exchanges $\left(\pi=(-1)^{J}\right)$, TRNI is possible only if the 
exchanged meson is charged. The isospin operator is the isovector $\left(\vec{\tau}_{1} \times \vec{\tau}_{2}\right)_{z}$. The spin-orbital structure is unique and generates only singlet-triplet transitions of the form ${ }^{1} P_{1} \leftrightarrow{ }^{3} P_{1},{ }^{1} D_{2} \leftrightarrow{ }^{3} D_{2}$, etc. Thus a vector meson exchange is completely defined by the meson mass $m_{v}$ Nuclear transitions should then be dominated by ${ }^{1} P_{1} \leftrightarrow{ }^{3} P_{1}$ transitions, which implies a factor of $\left(k_{F} / m_{v}\right)^{2}$ suppression relative to s-wave matrix elements of the TRI strong interaction, where $k_{F}$ is the Fermi momentum.

3) For $J \neq 0$ unnatural parity exchanges $\left(\pi=(-1)^{J+1}\right)$ all allowed TRNI PC partial wave amplitudes can contribute $\left({ }^{3} S_{1} \leftrightarrow{ }^{3} D_{1},{ }^{1} P_{1} \leftrightarrow{ }^{3} P_{1},{ }^{1} D_{2} \leftrightarrow{ }^{3} D_{2},{ }^{3} P_{2} \leftrightarrow\right.$ ${ }^{3} F_{2}$, etc. $)$ in combination with the isospin operators $1, \vec{\tau}_{1} \cdot \vec{\tau}_{2},\left(\vec{\tau}_{1}+\vec{\tau}_{2}\right)_{3},\left(\vec{\tau}_{1}-\vec{\tau}_{2}\right)_{3}$, and $\left(\vec{\tau}_{1} \otimes \vec{\tau}_{2}\right)_{3}^{2}$, depending on the isospin and G-parity of the exchanged meson. Thus $A_{1}$ exchange may be the simplest interaction producing isospin-conserving terms. Nuclear interactions should be dominated by the ${ }^{3} S_{1} \leftrightarrow{ }^{3} D_{1}$ and ${ }^{1} P_{1} \leftrightarrow{ }^{3} P_{1}$ amplitudes.

The interaction generated by $\rho^{ \pm}$exchange can be calculated from the vertices describing strong emission of a $\rho$ from one nucleon and TRNI reabsorption on another,

$$
\begin{gathered}
H^{\text {strong }}=g_{\rho} \bar{N}\left(\gamma_{\mu}+\frac{i \mu_{v}}{2 M} \sigma_{\mu \nu} q^{\nu}\right) \vec{\tau} \cdot \vec{\phi}_{\rho}^{\mu} N \\
H^{T R N I}=-\bar{g}_{\rho} g_{\rho} \bar{N} \frac{\mu_{v}}{2 M} \sigma_{\mu \nu} q^{\nu} \sqrt{2}\left(\tau_{-} \phi_{+}^{\mu *}-\tau_{+} \phi_{-}^{\mu *}\right) N
\end{gathered}
$$

The corresponding nonrelativistic TRNI PC $N N$ potential is

$$
V_{\rho}(\vec{r})=\frac{m_{\rho}^{3}}{M^{2}} g_{\rho}^{2} \bar{g}_{\rho} \frac{\mu_{v}}{4 \pi}(\tau(1) \times \tau(2))_{3} \frac{e^{-m_{\rho} r}}{m_{\rho}^{3} r^{3}}\left(1+m_{\rho} r\right)\left(\vec{\sigma}_{1}-\vec{\sigma}_{2}\right) \cdot \vec{\ell}
$$

where $m_{\rho}=770 \mathrm{MeV}, g_{\rho}=2.79, \mu_{v}=3.70$, and $\bar{g}_{\rho}$ parameterizes the strength of the TRNI vertex. The $N N$ separation is $\vec{r}=\vec{r}_{1}-\vec{r}_{2}$ and $\vec{\ell}=\vec{r} \times \frac{1}{2}\left(\vec{p}_{1}-\vec{p}_{2}\right)$. This potential, first derived by Simonius ${ }^{5}$ ), is the longest ranged TRNI PC $N N$ mesonexchange interaction.

A TRNI PC $N N$ interaction arising from an unnatural parity exchange, the $A_{1}(1260)$, was introduced by Sudarshan ${ }^{21}$ ) and discussed by Binstock, Bryan, and Gersten and also by Simonius and Wyler ${ }^{22}$ ). 


\section{Long-ranged Contributions to Atomic Electric Dipole Moments}

TRNI PC interactions in concert with the weak interaction can generate TRNI PNC observables. It has been appreciated for some time that the stringent limits existing on edms must then impose interesting limits on TRNI PC interactions. For instance, while detailed calculations do not appear to have been carried out, dimensional arguments have been made that the bounds on the neutron edm must limit meson-nucleon TRNI PC couplings to $\lesssim 10^{-4} \cdot{ }^{4,23}$ )

Experimental searches for the edms of neutral atoms have progressed very rapidly in recent years. The results have been used as bounds on the edms of the electron, proton, and neutron and as constraints on possible TRNI PNC $N N$ and $e N$ interactions. We have begun a study of the implications of such measurements for TRNI PC mesonnucleon couplings, that is, on long-ranged interactions that might contribute through weak radiative corrections to atomic edms. An investigation of short-ranged radiative mechanisms was recently completed by Khriplovich ${ }^{24}$ ) and Conti and Khriplovich ${ }^{25}$ ).

Below we examine the atomic edm that is generated by $Z$ exchange between the atom's electrons and a nucleus in which a TRNI PC interaction operates. One interesting aspect of this mechanism is that the induced edm is truly an atomic quantity: there is no coupling of the external electric field directly to the nucleus (see Fig. 1a). This contrasts with the situation where the nucleus is the source of the edm, as shown in Fig. 1b. Then, as discussed by Schiff ${ }^{26}$ ), the nucleus is almost completely shielded by the response of the atomic electrons to the external perturbation. The shielding is incomplete because of the finite extent of the nucleus. The penetration effects that account for the residual interaction grow rapidly with the atomic charge $Z$. Thus measurements of edms in high- $Z$ atoms have easily provided the best constraints on nuclear TRNI PNC interactions. In contrast, for the process shown in Fig. 1a, there are no large shielding effects to diminish the usefulness of constraints from light atoms. 
We are interested in defining a limit on $\alpha$, the ratio of typical TRNI PC matrix elements to those of the strong interaction. Adopting the Simonius $\rho$-exchange potential as a representative meson-nucleon interaction, we begin by determining the atomic edm limits on $\bar{g}_{\rho}$.

The coupling of the exchange $Z$ to the nucleus must be TRNI and PC. Groundstate expectation values linear in the TRNI amplitude will occur for odd multipoles of the axial charge operator $\left(C 1^{5}, C 3^{5}\right.$, etc.), even magnetic multipoles of the axial three-current $\left(M 2^{5}, M 4^{5}\right.$, etc.), and even electric multipoles of the vector three-current (E2, E4, etc.). The electric multipoles vanish in leading order by Siegert's theorem. Furthermore, the contributions of higher multipoles to the electron-nucleus interaction are suppressed by powers of $\left(r_{N} / r_{A}\right)$ where $r_{N}$ and $r_{A}$ are the nuclear and atomic radii: large angular momentum transfers produce unfavorable electron overlaps with the nucleus. One concludes that the important contribution to $Z$ exchange is the dipole term $C 1_{e} \cdot C 1_{N}^{5}$. Thus we need only consider the interaction

$$
H=\frac{G}{\sqrt{2}} \int d \vec{x} \rho_{e}^{V}(\vec{x}) \rho_{N}^{5}(\vec{x})
$$

where $\rho_{e}^{V}(\vec{x})$ in the electron vector charge operator and $\rho_{N}^{5}(\vec{x})$ the nuclear axial charge. For nonrelativistic nucleons the corresponding electron potential is

$$
\begin{aligned}
H(\vec{x})= & \frac{G}{\sqrt{2}} \frac{1}{4 M} g_{A}\left(1-4 \sin ^{2} \theta_{w}\right) \rho_{e}^{V}(\vec{x}) \\
& <\phi^{g s}\left|\sum_{i=1}^{A}\left[\delta\left(\vec{x}-\vec{x}_{i}\right) \frac{1}{i} \vec{\sigma}_{i} \cdot \vec{\nabla}_{i}-\frac{1}{i} \vec{\sigma}_{i} \cdot \bar{\nabla}_{i} \delta\left(\vec{x}-\vec{x}_{i}\right)\right] \tau_{3}(i)\right| \phi^{g s}>
\end{aligned}
$$

where $g_{A}=1.25$ and $\mid \phi^{g s}>$ is the nuclear ground state.

The nuclear matrix element in Eq. (10) will be proportional to $\bar{g}_{\rho}$. It can be evaluated in first-order perturbation theory by calculating the nuclear polarizability associated with $V_{\rho}(\vec{r})$. There are elegant Green's function techniques for accomplishing 
this ${ }^{27}$ ). Here we take a simpler approach, but one that is still likely to be reasonably accurate while providing a clearer picture of the underlying physics.

If $\left|\phi_{0}^{g s}\right\rangle$ is the unperturbed TRI ground state, the nuclear matrix element in Eq. (10) can be written

$$
\sum_{n}<\phi_{0}^{g s}\left|V_{\rho} \frac{\left|I_{n}><I_{n}\right|}{E_{0}-E_{I_{n}}} \rho_{N}^{5}(\vec{x})\right| \phi_{0}^{g s}>+h . c .
$$

where the sum extends over a complete set of excited states. The important $C 1^{5}$ projection of $\rho_{N}^{5}(\vec{x})$ carries the quantum numbers of the Gamow-Teller operator. Experimentally it is known that most of the GT strength resides in a broad resonance at an excitation energy of about $\hbar \omega$, the shell splitting. Thus we approximate the energy denominators by $\hbar \omega$ and complete the sum by closure to find

$$
-\frac{1}{\hbar \omega}<\phi_{0}^{g s}\left|V_{\rho} \rho_{N}^{5}(\vec{x})+\rho_{N}^{5}(\vec{x}) V_{\rho}\right| \phi_{0}^{g s}>
$$

The operator in Eq. (12) is three-body. We borrow a frequently-used trick from studies of nuclear PNC and replace this operator by an effective one-body one, defined in this case by

$$
\begin{aligned}
& \sum_{\alpha \beta}<\alpha\left|0_{e f f}^{(1)}(\vec{x})\right| \beta>a_{\alpha}^{+} a_{\beta}= \\
& {\left[\frac{1}{2} \sum_{\alpha_{1} \alpha_{2} \beta_{1} \beta_{2}}<\alpha_{1} \alpha_{2}\left|V_{\rho}\right| \beta_{2} \beta_{1}>a_{\alpha_{1}}^{+} a_{\alpha_{2}}^{+} a_{\beta_{1}} a_{\beta_{2}} \sum_{\alpha_{3} \beta_{3}}<\alpha_{3}\left|\rho_{N}^{5}(\vec{x})\right| \beta_{3}>a_{\alpha_{3}}^{+} a_{\beta_{3}}\right]_{\alpha \beta}^{\substack{\text { doubiy } \\
\text { contracted }}} .}
\end{aligned}
$$

That is, we sum over all terms on the right-hand side containing two pairs of identical $\alpha$ s and $\beta$ s. Such approximations tend to be quite accurate in heavy nuclei because most of the strength of the operator is generated by coherent diagonal sums over deeply bound nucleons. It is especially appropriate to the present application because the two-body operator $V_{\rho}(\vec{r})$ vanishes in the one-body approximation for spin-symmetric 
nuclear matter. Thus we expect the important contributions to Eq. (12) to arise from spin correlations between $V_{\rho}$ and $\rho_{N}^{5}(\vec{x})$ which the averaging technique ought to isolate.

We performed the averaging in a spin- and isospin-symmetric Fermi gas. Three properties of the operators $V_{\rho}$ and $\rho_{N}^{5}(\vec{x})$ permit only a single contraction to survive:

1) $\rho_{N}^{5}(\vec{x})$ has no diagonal single-particle matrix elements because of its properties under time reversal.

2) As $V_{\rho}(\vec{r})$ vanishes when it is separately averaged, all tadp ies at the $\rho$ vertices vanish.

3) Since $\rho_{N}^{5}(\vec{x})$ is neutral while $V_{\rho}(\vec{r})$ carries charge, there are no polarization diagrams (i.e., diagrams where $\rho_{N}^{5}(\vec{x})$ and $V_{\rho}(\vec{r})$ enter the opposite ends of a particlehole bubble). The surviving term is

$$
-\sum_{\substack{\alpha_{1}<F \\ \beta_{1}>F}}<\alpha \alpha_{1}\left|V_{\rho}\right| \beta_{1} \beta><\beta_{1}\left|\rho_{N}^{5}(\vec{x})\right| \alpha_{1}>
$$

shown in Fig. 2 (and the time-reversed diagram).

The evaluation of the integrals in Eq. (14) is tedious but not difficult. We simplified the calculation by ignoring the momentum in the $\rho$ propagator, thereby approximating the exchange by a contact interaction. One expects this to introduce errors on the order of $\left(k_{F} / m_{\rho}^{2}\right)$. (A recent shell-model study ${ }^{28}$ ) of this approximation, which has been used frequently in studies of PNC since the work of $\mathrm{Michel}^{29}$ ), found errors of $5 \%$ in the estimates of uncor related matrix elements of a rho-exchange TRNI PNC potential.) We find

$$
\begin{aligned}
<\alpha\left|0_{e f f}^{(1)}(\vec{x})\right| \beta>= & -\frac{4 i}{15 \pi^{4}} \frac{1}{\hbar \omega} \frac{k_{F}^{5}}{m_{\rho}^{2} M^{2}} g_{\rho}^{2} \bar{g}_{\rho} \frac{\mu_{v}}{4 \pi} \\
& e^{i\left(\vec{k}_{\beta}-\vec{k}_{\alpha}\right) \cdot \vec{x}}<m_{\tau_{\alpha}}\left|m_{\tau_{\beta}}>\left(\vec{k}_{\alpha}-\vec{k}_{\beta}\right)<m_{\alpha}\right| \vec{\sigma} \mid m_{\beta}>W\left(\frac{\left|\vec{k}_{\alpha}-\vec{k}_{\beta}\right|}{k_{F}}\right)
\end{aligned}
$$


where

$$
W(z)=\left\{\begin{array}{cc}
1, & z \geq 2 \\
-\frac{17}{256} z^{5}+\frac{25}{32} z^{3}-\frac{5}{4} z^{2}+\frac{15}{16} z, & z<2
\end{array}\right.
$$

This "form factor" represents the results of averaging the nonlocal operator of Fig. 2 over nuclear densities. Despite its complicated form, $W(z)$ is very well approximated by $0.5 z$ for typical momentum transfers $\left(\lesssim 2 k_{F}\right.$ ). If we use 0.5 as an average value we can define the first-quantized operator

$$
O_{e f f}^{(1)}(\vec{x})=A \frac{k_{F}^{5}}{m_{\rho}^{2} M^{2}} \frac{1}{\hbar \omega} \sum_{i=1}^{A} \vec{\sigma}_{i} \cdot \vec{\nabla}_{i} \delta\left(\vec{x}-\vec{x}_{i}\right)
$$

where $A=-\frac{16}{15 \pi} g_{\rho}^{2} \bar{g}_{\rho} \frac{\mu_{v}}{4 \pi}$.

The corresponding Hamiltonian for the atomic electrons is

$$
\left.H=\frac{G}{\sqrt{2}} \frac{1}{4 M} g_{A}\left(1-4 \sin ^{2} \theta_{w}\right) A \frac{k_{F}^{5}}{m_{\rho}^{2} M^{2}} \frac{1}{\hbar \omega}<\phi_{0}^{g a}\left|\sum_{i=1}^{A} \vec{\sigma}_{i} \cdot \vec{\nabla}_{i} \rho_{e}\left(\vec{x}_{i}\right)\right| \phi_{0}^{g \Delta}\right\rangle .
$$

Ignoring variations of the electron wave functions over the nucleus, one finds an effective TRNI PNC atomic potential

$$
H(\vec{x})=-\frac{G}{\sqrt{2}} \frac{1}{2 M} g_{A}\left(1-4 \sin ^{2} \theta_{w}\right) A \frac{k_{F}^{5}}{m_{\rho}^{2} M^{2}} \frac{1}{\hbar \omega}<\vec{I}>\cdot \vec{\nabla} \delta(\vec{x})
$$

where $\vec{I}$ is the nuclear spin. While we have made some simplifications in this derivation, the final result exhibits no dependence on the detailed nuclear structure.

This atomic potential is identical to that for electrons interacting with a nuclear edm, once the effects of shielding have been included ${ }^{30}$ )

$$
H_{e d m}(\vec{x})=-4 \pi e Q \frac{\langle\vec{I} \geq}{I} \cdot \vec{\nabla} \delta(\vec{x})
$$

This result was obvious from the outset: both interactions are contact ones and both have the multipole character $C 1_{e} \cdot C 1_{N}^{5}$. The difference between Eqs. (18) and (19) is the replacement of the Schiff moment $Q$, which depends on nuclear structure and 
varies rapidly with $Z$ for fixed weak parameters, by a quantity that depends only on coupling constants and the Fermi momentum. One concludes that good atomic edm experiments in light atoms, such as ${ }^{3} \mathrm{He}^{31}$ ), could provide important constraints on TRNI PC interactions, even if such experiments are not competitive as tests of nuclear edms.

We can now exploit the atomic structure calculations ${ }^{32,33}$ ) that have been done to extract $Q$ from the experimental data. The most restrictive limits on Schiff moments come from the experiments on ${ }^{199} \mathrm{Hg}^{34}$ ) and $\mathrm{TlF}{ }^{35}$ )

$$
\begin{aligned}
Q\left({ }^{199} \mathrm{Hg}\right) & =(-1.8 \pm 3.8) \cdot 10^{-10} e f \\
Q\left({ }^{205} \mathrm{Tl}\right) & =(-1.8 \pm 3.0) \cdot 10^{-10} e f
\end{aligned}
$$

where $e^{2}=\alpha$. Using $\sin ^{2} \theta_{W}=0.22, k_{F}=280 \mathrm{MeV}$, and $\hbar \omega=41 A^{-1 / 3} \mathrm{MeV} \sim 7$ $\mathrm{MeV}$, we find

$$
\left|\bar{g}_{\rho}\right|^{e f f} \lesssim \begin{cases}0.018 \pm 0.038, & { }^{199} \mathrm{Hg} \\ 0.018 \pm 0.029, & { }^{205} \mathrm{Tl}\end{cases}
$$

The notation "eff" reminds us that this is the appropriate coupling constant to use in nuclear calculations where, as in the above Fermi gas example, no short-ranged $N N$ correlation are included. The bounds on the true meson-nucleon coupling $\bar{g}_{\rho}$ are weaker than those of Eq. (20).

The final step is relating this limit to a bound on $\alpha$. We first evaluate the $N N$ matrix elements of $V_{\rho}(\vec{r})$ for harmonic oscillator wave functions, using parameters and shells typical of the proton and neutron Fermi seas near $A=200: b=2.45 f$ and the $2 \mathrm{~d}_{3 / 2}, 1 \mathrm{~h}_{11 / 2}, 1 \mathrm{~h}_{9 / 2}, 3 \mathrm{p}_{1 / 2}$, and $1 \mathrm{i}_{13 / 2}$ shells. Within this space there are 41 nonzero pn matrix elements of $V_{\rho}(\vec{r})$ for two-particle angular momenta less than 5 . From these we calculate a rms TRNI PC matrix element of $10.3 \mathrm{keV}$ when $\bar{g}_{\rho}^{\text {eff }}=1$. Again, as we include no short-ranged correlations in the evaluation, $\bar{g}_{\rho}^{e f f}$ is the proper parameter. The small value we find is consistent with the general arguments in Section 3: the 
potential is short-ranged yet must connect relative $p$-states. Thus the choice $\bar{g}_{\rho}^{\text {eff }}=1$ produces TRNI PC matrix elements that are already smaller than the limits set by detailed balance experiments with direct interactions ${ }^{36}$ ).

We then derived a corresponding strong interaction rms matrix element for the same 41 transitions, using two well-known shell model residual interactions, SerberYukawa and Rosenfeld ${ }^{37}$ ). The values are $197 \mathrm{keV}$ and $400 \mathrm{keV}$. Thus we take $300 \mathrm{keV}$ as the scale of strong interaction matrix elements. A bound on $\alpha$, the ratio of the $N N$ matrix elements of the TRNI PC and strong interactions, then follows from Eq. (20)

$$
|\alpha| \lesssim(0.6 \pm 1.0) \cdot 10^{-3}
$$

This is a value quite similar to those obtained in the CN studies summarized in Section 2. New atomic edm experiments will soon improve this limit by an order of magnitude. [Incidentally, it is not clear to us that the CN limits apply with equal weight to TRNI PC interactions that violate isospin, as does $V_{\rho}(\vec{r})$. As we noted in Section 3, most of the possible TRNI PC amplitudes do break isospin. The $A_{1}$ potential used by Simonius and $\mathrm{Wyler}^{22}$ ) is isoscalar, however, so a valid comparison with the CN results could certainly be made in that case. The atomic edm constraints on the $A_{1}$ potential would yield a bound very like that of Eq. (21).]

\section{Conclusions}

The arguments above provide one bound on the strength of long-distance (i.e., meson-exchange) TRNI PC interactions. Such meson-exchange interactions will also generate other contributions to atomic edms: in combination with the hadronic weak interaction, TRNI PC interactions will generate nucleon edms through meson loops and nuclear edms through nuclear polarizabilities and through $\rho \pi \gamma$ and $\rho \rho \gamma$ exchange currents. These will be discussed elsewhere ${ }^{7}$ ). We know that the net result will be bounds no worse than Eq. (21). 
Limits on meson-nucleon couplings from such studies are easily connected to the physics probed in compound nucleus TRNI tests. Atomic edm limits are improving rapidly. At the same time there are new ideas for $\mathrm{CN}$ tests, such as the possibility of exploiting the five-fold correlation for epithermal neutrons, that may yield substantial improvements, too. So, while it is difficult to guess which approach will eventually prove more productive, it is clear that the constraints on $\alpha$ will continue to improve.

However both sets of results appear quite modest from other perspectives. Khriplovich $^{24}$ ) has recently considered one-loop weak radiative corrections to TRNI PC interactions arising from short-ranged mechanisms, i.e., length scales associated with weak boson masses. While much of the analysis is dimensional, he concludes that edms limit the quark-quark TRNI PC interaction strength to $\lesssim 10 G m_{\pi}^{2}$, and perhaps the corresponding $N N$ potential strength to $\lesssim 100 G m_{\pi}^{2} \sim 10^{-5}$. Conti and Khriplovich ${ }^{25}$ ) considered two-loop contributions and concluded that such limits could be further tightened, perhaps by five orders of magnitude. Finally we recall the theorem of Herczeg, Kambor, Simonius, and $\mathrm{Wyler}^{2}$ ) that a general feature of renormalizable gauge theories with elementary quarks is the absence of flavor-conserving TRNI PC interactions until second order in the boson-fermion couplings. Thus $10^{-16}$ may be relevant scale for such interactions.

We thank Peter Herczeg, Daniel Wyler, David Bowman, Chris Gould, and Steve Pollock for helpful discussions. One of us (WH) also thanks Bruce McKellar and the University of Melbourne for their hospitality while this work was completed. This research was supported in part by the Department of Energy and by the National Science Foundation US-Australia Cooperative Program. 


\section{References}

1) For general reviews see P. Herczeg, in Tests of Time Reversal Invariance in Neutron Physics, ed. N.R. Roberson, C.R. Gould, and J.D. Bowman (World Scientific, Singapore, 1987), p. 24;

E.M. Henley, ibid., p. 1

2) P. Herczeg, J. Kambor, M. Simonius, and D. Wyler, "Parity Conserving Time Reversal Violation in Flavor Conserving Quark-Quark Interactions," to be published; Reported by P. Herczeg, Hyp. Int. 75 (1992) 127

3) E.P. Wigner, SIAM Rev. 9 (1967) 1

4) L. Wolfenstein, Nucl. Phys. B77 (1974) 375

5) M. Simonius, Phys. Lett. 58B (1975) 147

6) P. Herczeg, Nucl. Phys. 75 (1966) 655

7) W.C. Haxton, Antje Höring, and M. Musolf, in preparation

8) E. Blanke, H. Driller, W. Glockle, H.Genz, A. Richter, and G. Schrieder, Phys. Rev. Lett. 51 (1983) 355;

A. Richter, in Interaction Studies in Nuclei, ed. H. Jochim and B. Ziegler (NorthHolland, Amsterdam, 1975) p. 191

9) D. Boosé, H.L. Harney, and H.A. Weidenmüller, Phys. Rev. Lett. 56 (1986) 2012; Z. Phys. A325 (1986) 363

10) J.B. French, V.K. Kota, A. Pandey, and S.Tomsovic, Phys. Rev. Lett. 54 (1985) 2313; Ann. Phys. 181 (1988) 198

11) R.U. Haq, A. Pandey, and O. Bohigas, Phys. Rev. Lett. 48 (1982) 1086

12) J.B. French, V.K. B. Kota, A. Pandey, and S. Tomsovic, Ann. Phys. 181 (1988) 235

13) P.K. Kabir, in Proc. Workshop on Fundamental Interactions with Neutrons, NBS Special Publication 711, ed. G.L. Greene (US Department of Commerce, 1985) 
14) V.G. Baryshevsky, Yad. Fiz. 38 (1983) 1162 [Sov. J. Nucl. Phys. 38 (1983) 699]

15) V.E. Bunakov, Phys. Rev. Lett. 60 (1988) 2250

16) A.L. Barabanov, Yad. Fiz. 44 (1986) 1163 [Sov. J. Nucl. Phys. 44 (1986) 755]

17) V.E. Bunakov and V.P. Gudkov, in Tests of Time Reversal Invariance in Neutron Physics, ed. N.R. Roberson, C.R. Gould, and J.D: Bowman (World Scientific, Singapore, 1987), p. 175

18) V.E. Bunakov, E.D. Davis, and H.A. Weidenmüller, Phys. Rev. C42 (1990) 1718

19) L. Stodolsky, Phys. Lett. B172 (1986) 5;

P. K. Kabir, Phys. Rev. D77 (1988) 1856

20) J.E. Koster et al., Phys. Lett. B267 (1991) 23

21) E.C.G. Sudarshan, Proc. Roy. Soc. A305 (1968) 375

22) R. Bryan and A. Gersten, Phys. Rev. Lett. 26 (1971) 1000 and 27 (1971) 1102;

J. Binstock, R. Bryan, and A. Gersten, Phys. Lett. 48B (1974) 77;

M. Simonius and D. Wyler, Nucl. Phys. A286 (1977) 182

23) P. Herczeg, Hyp. Int. 75 (1992) 127

24) I.B. Khriplovich, Nucl. Phys. B352 (1991) 385

25) R.S. Conti and I.B. Khriplovich, Phys. Rev. Lett. 68 (1992) 3262

26) L.I. Schiff, Phys. Rev. 132 (1963) 2194

27) W.C. Haxton, to appear in Proc. Intl. Conf. on Nuclear Structure at High Angular Momentum, Ottawa, May 1992.

28) A. Griffiths and P. Vogel, Caltech preprint (1992)

29) F.C. Michel, Phys. Rev. 133 (1964) B329

30) See, for example, O.P. Sushkov, V.V. Flambaum, and I.B. Khriplovich, Zh. Eksp. Teor. Fiz. (JETP) 87 (1984) 1521

31) W. Fairbank's interest in mounting a high-precision measurement of the atomic edm of ${ }^{3} \mathrm{He}$ is said to have motivated Schiff's [26] study 
32) V.V. Flambaum, I.B. Khriplovich, and O.P. Sushkov, Nucl. Phys. A449 (1986) 750

33) P.V. Coveney and P.G.H. Sandars, J. Phys. B16 (1983) 3727

34) S.K. Lamoreaux, J.P. Jacobs, B.R. Heckel, F.J. Raab, and N. Fortson, Phys. Rev. Lett. 59 (1987) 2275

35) P. Cho, K. Sangster, and E.A. Hinds, Phys. Rev. Lett. 63 (1989) 2559

36) P.A. Moldauer, Phys. Lett. B26 (1968) 713

37) See, for example, Amos deShalit and Herman Feshbach, Theoretical Nuclear Physics, vol. 1 (John Wiley and Sons, New York, 1974), p. 373 


\section{Figure Captions}

Fig. 1 Interaction of an external field with an atomic edm produced by a TRNI PC interaction in the nucleus combined with $Z$ exchange between the nucleus and the atomic electrons (a). The corresponding diagram for the interaction of an external field with the edm residing in the nucleus is shown in (b). In this case the atomic polarizability cancels the direct coupling in the limit of a point nucleus.

Fig. 2 The one-body effective operator representing the coupling of the axial charge to a nucleus in which a TRNI PC interaction operates. 
(a)

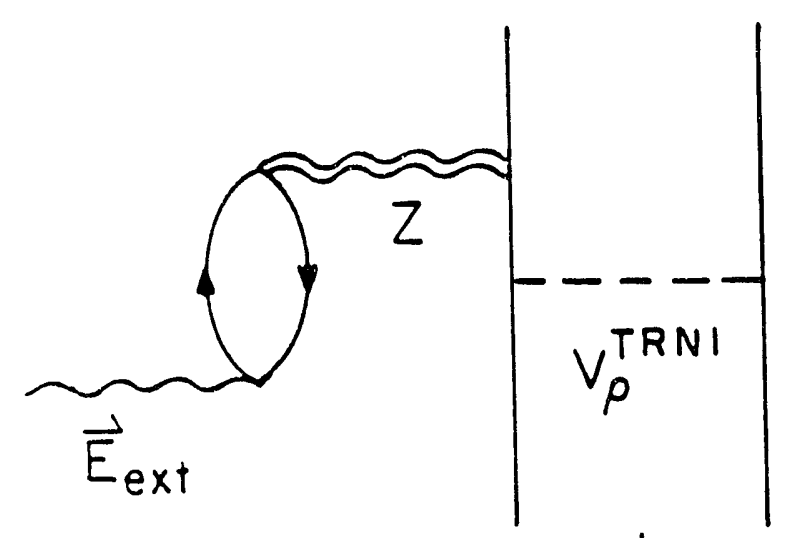

(b)

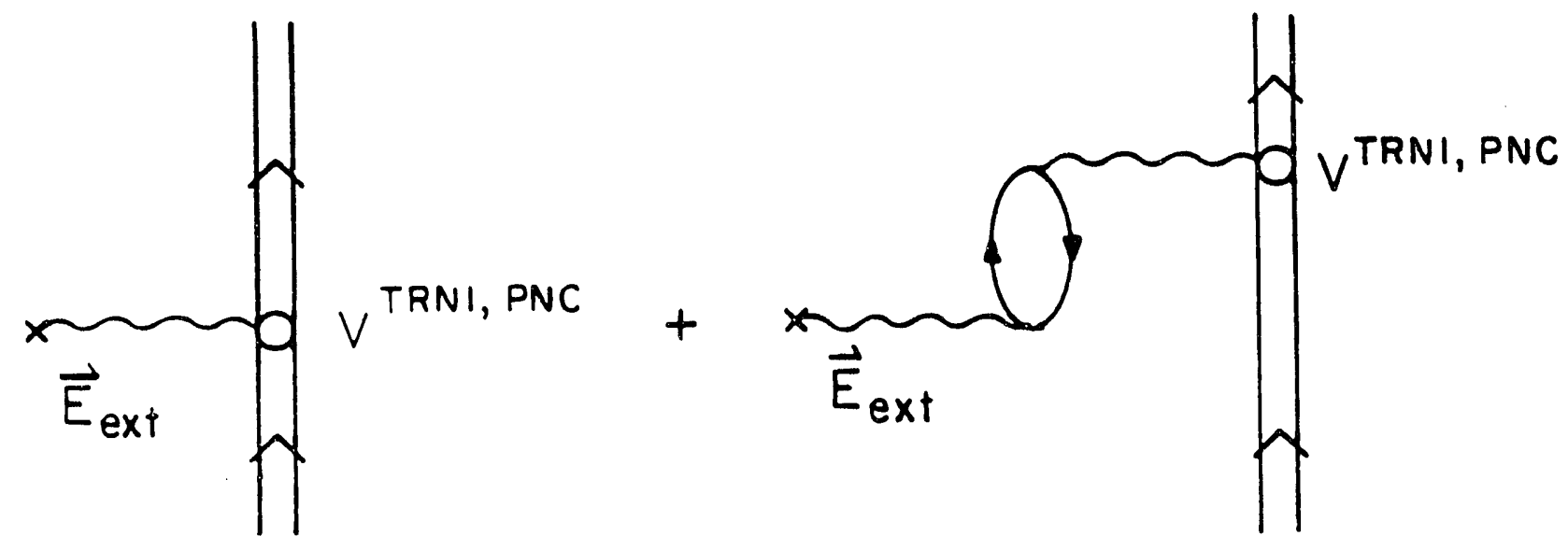




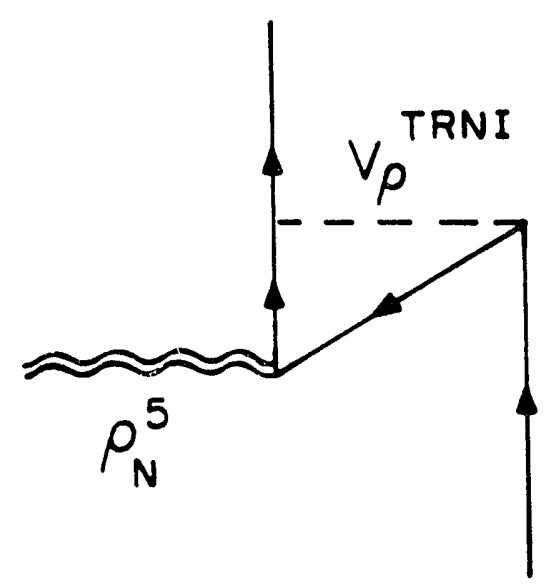

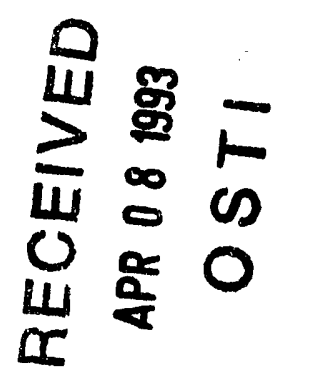

Figure 2 

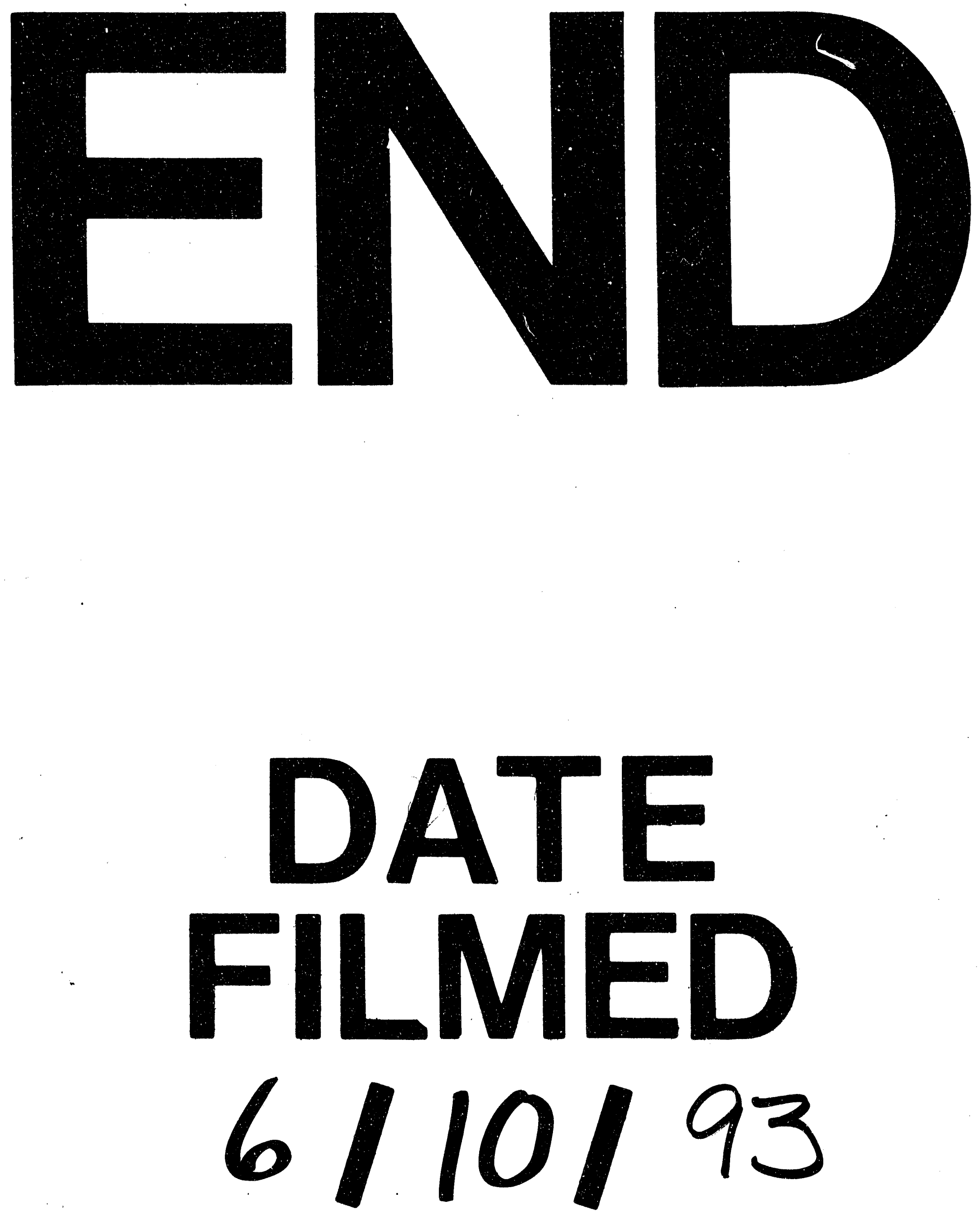
